\title{
$V$-Invariant Methods for Generalised Least Squares Problems
}

\author{
M.R. Osborne \\ Mathematical Sciences Institute, Australian National University, ACT 0200, Australia
}

\begin{abstract}
An important consideration in solving generalised least squares problems is the dimension of the covariance matrix $V$. This has the dimension of the data set and is large when the data set is large. In addition the problem can be formulated to have a well determined solution in cases where $V$ is illconditioned or singular, a class of problems that includes the case of equality constrained least squares. This paper considers a class of methods which factorize the design matrix $A$ while leaving $V$ invariant, and which can be expected to be well behaved exactly when the original problem solution is well behaved. Implementation is most satisfactory when $V$ is diagonal. This can be achieved by a preprocessing step in which $V$ is replaced by the diagonal matrix $D$ which results from the modified Cholesky factorization $P V P^{T} \rightarrow L D L^{T}$ where $L$ is unit lower triangular and $P$ is the permutation matrix associated with diagonal pivoting. Conditions under which this replacement is satisfactory are investigated.
\end{abstract}

\section{Introduction}

The generalised least squares problem is

$$
\min _{\mathbf{x}, \mathbf{r}} \mathbf{r}^{T} V^{-1} \mathbf{r} ; \mathbf{r}=A \mathbf{x}-\mathbf{b}
$$

where $A \in R^{p} \rightarrow R^{n}, \operatorname{rank}(A)=p<n, \mathbf{x} \in R^{p}, \mathbf{r} \in R^{n}$. In this form it requires $V$ to be positive definite and hence invertible. However, the necessary conditions for this problem to have a solution can be cast in the form

$$
\left[\begin{array}{cc}
V & -A \\
-A^{T} & 0
\end{array}\right]\left[\begin{array}{l}
\boldsymbol{\lambda} \\
\mathbf{x}
\end{array}\right]=\left[\begin{array}{c}
-\mathbf{b} \\
0
\end{array}\right]
$$

where $\lambda$ is the vector of Lagrange multipliers and is related to the residual vector by $\mathbf{r}=V \boldsymbol{\lambda}$. This proves to have solutions under weaker conditions on $V$.

In (2) the solution of the generalised least squares problem requires the solution of a linear system with symmetric, indefinite matrix. This poses problems in the data analytic context because $n$ may well need to be large as a consequence of the generic $n^{-1 / 2}$ rate of convergence in stochastic estimation problems, while $p$ has to be small in typical regression situations. Thus the size of $V$ is potentially the main source of difficulty in otherwise well behaved problems. This would also suggest that if structure is present in $V$ then advantage should be taken of this fact. One possible approach is considered here based on the paper [2]. The generalisation embodied in the formulation 
(2) has the interesting advantage that it permits least squares problems subject to linear equality constraints to be considered in the same framework as the generalised least squares problem. Let the constrained problem be written

$$
\min _{\mathbf{x}, \mathbf{r}_{2}} \mathbf{r}_{2}^{T} V^{-1} \mathbf{r}_{2} ; \mathbf{r}_{2}=A \mathbf{x}-\mathbf{b}, 0=C \mathbf{x}-\mathbf{d}
$$

where $C \in R^{p} \rightarrow R^{m}, m<p$, is assumed to have full rank. This ensures that the constraints are consistent for arbitrary problem data $\mathbf{d}$. One possible approach involves reformulating the problem using penalised least squares:

$$
\min _{\mathbf{x}, \mathbf{r}}\left\{\mathbf{r}_{2}^{T} V^{-1} \mathbf{r}_{2}+\sum_{i=1}^{m} \sigma^{2} W_{i}^{-2}\left(\mathbf{r}_{1}\right)_{i}^{2}\right\}
$$

where $\mathbf{r}_{1}=C \mathbf{x}-\mathbf{d}$, and $W=\operatorname{diag}\left\{W_{i}\right\}$ is some bounded positive scaling matrix. The idea is that as $\sigma$ gets large the penalised term will dominate unless $\left\|\mathbf{r}_{1}\right\| \rightarrow 0$. It is straightforward to show a convergence rate of $O\left(\sigma^{-2}\right)$. But (3) is just a generalised least squares problem and can be reformulated as

$$
\min _{\mathbf{x}, \mathbf{s}} \mathbf{s}^{T} \mathbf{s} ;\left[\begin{array}{ll}
\sigma^{-1} W & \\
& L
\end{array}\right] \mathbf{s}=\left[\begin{array}{l}
C \\
A
\end{array}\right] \mathbf{x}-\left[\begin{array}{l}
\mathbf{d} \\
\mathbf{b}
\end{array}\right] .
$$

This problem has the limiting form as $\sigma \rightarrow \infty$

$$
\min _{\mathbf{x}, \mathbf{s}} \mathbf{s}^{T} \mathbf{s} ;\left[\begin{array}{ll}
0 & \\
& L
\end{array}\right] \mathbf{s}=\left[\begin{array}{l}
C \\
A
\end{array}\right] \mathbf{x}-\left[\begin{array}{l}
\mathbf{d} \\
\mathbf{b}
\end{array}\right] .
$$

The solution of this problem is well determined provided

$$
M=\left[\begin{array}{cc}
{\left[\begin{array}{ll}
0 & 0 \\
0 & V
\end{array}\right]} & -\left[\begin{array}{l}
C \\
A
\end{array}\right] \\
-\left[\begin{array}{ll}
C^{T} & A^{T}
\end{array}\right] & 0
\end{array}\right]
$$

is nonsingular. The ordering of terms here proves important.

The development of this paper is as follows. The next two sections consider $V$ invariance and its application to the factorization of the design matrix $A$ with only minor differences from [2]. Practical implementation consideration puts an emphasis on diagonal $V$ with elements ordered in increasing size, and it is suggested [4] that the $L D L^{T}, L$ lower triangular with unit diagonal, modification of the basic Cholesky factorization of $V$ using diagonal pivoting [3] could prove useful in weakening this requirement. The major contribution in this paper is made in the concluding section where it is argued that the error that occurs when small elements in the computed $D$ are set to zero is, with some qualification, of the same size as these small elements. In fact this argument extends to the case where $D$ supports multiple scales switching between these in discrete steps. Also, it is not required that all steps of the Cholesky factorization be completed successfully in the case that $V$ is (almost) semi-definite. It is shown that one consequence of the ability to support multiple scales is a requirement for column pivoting in the factorization of the design matrix $A$, especially when equality constraints are present. 


\section{$2 \quad V$-Invariance}

The motivation for introducing algorithms for the solution of generalised least squares problems based on transformations with special invariance properties derives from the special case of the methods for the linear least squares problem based on orthogonal factorization of the design matrix $A$ where an orthogonal matrix $Q$ is constructed to reduce $A$ to upper triangular form while preserving column lengths corresponding to $V=I$. The condition for $V$-invariance generalises this metric condition to

$$
J V J^{T}=V
$$

Now assume that a $V$-invariant factorization of $A$ is given by

$$
J A=\left[\begin{array}{l}
U \\
0
\end{array}\right],
$$

and that $V$ has the form

$$
V=\left[\begin{array}{cc}
O_{k} & 0 \\
0 & V_{2}
\end{array}\right]
$$

where $O_{k}$ is the $k \times k$ zero matrix and $V_{2}$ has the form

$$
V_{2}=\left[\begin{array}{ll}
V_{11} & V_{12} \\
V_{21} & V_{22}
\end{array}\right]
$$

where $V_{11} \in R^{p-k} \rightarrow R^{p-k}$. Then the transformation applied to equation (2) gives

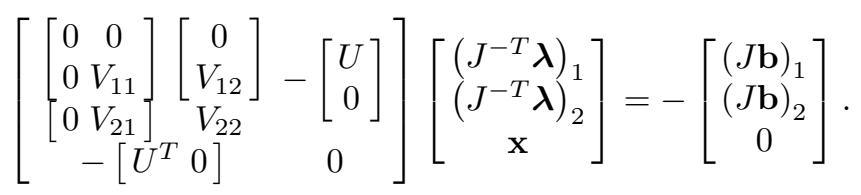

This system reduces to the equations

$$
\begin{aligned}
U^{T}\left(J^{-T} \boldsymbol{\lambda}\right)_{1} & =0 \\
V_{22}\left(J^{-T} \boldsymbol{\lambda}\right)_{2} & =-(J \mathbf{b})_{2} \\
{\left[\begin{array}{c}
0 \\
V_{12}
\end{array}\right]\left(J^{-T} \boldsymbol{\lambda}\right)_{2}-U \mathbf{x} } & =-(J \mathbf{b})_{1} .
\end{aligned}
$$

Remark 1. If $U$ in (4) is nonsingular, and $V_{2}$ is ordered so that $V_{22}$ is nonsingular, then the generalised least squares problem has a well determined solution.

To construct factorizations it is convenient to develop elementary $V$-invariant transformations. Let

$$
J=I-2 \mathbf{u v}^{T}
$$


If $\mathbf{v}^{T} V \mathbf{v}>0$ then the condition of $\mathrm{V}$-invariance determines $\mathbf{u}$ :

$$
\mathbf{u}=\frac{1}{\mathbf{v}^{T} V \mathbf{v}} V \mathbf{v}, J=I-\frac{2}{\mathbf{v}^{T} V \mathbf{v}} V \mathbf{v} \mathbf{v}^{T} .
$$

In this case $\mathbf{u}^{T} \mathbf{v}=1$ so that $J$ is also an elementary reflector $\left(J^{2}=I\right)$. If $\mathbf{v}=$ $\left[\begin{array}{c}\mathbf{v}_{1} \\ 0\end{array}\right], \mathbf{v}_{1} \in R^{k}$ then $\mathbf{v}^{T} V=0$ and the above derivation of $J$ breaks down. A $V$ invariant transformation is given by

$$
J=I-2\left[\begin{array}{l}
\mathbf{u}_{1} \\
\mathbf{u}_{2}
\end{array}\right]\left[\begin{array}{ll}
\mathbf{v}_{1}^{T} & 0
\end{array}\right],
$$

and $J^{2}=I$ if $\mathbf{v}_{1}^{T} \mathbf{u}_{1}=1$. There is a useful connection between the two forms of transformations. Let

$$
V_{\varepsilon}=\left[\begin{array}{ll}
\varepsilon W & \\
& V_{2}
\end{array}\right]
$$

where $V_{\varepsilon} \in R^{k} \rightarrow R^{k}$ is a positive diagonal matrix. Then the $V_{\varepsilon}$-invariant transformation derived from (7) but written in terms of $\mathbf{u}$ is

$$
\begin{aligned}
J_{\varepsilon} & =I-\frac{2}{\mathbf{u}^{T} V_{\varepsilon}^{-1} \mathbf{u}} \mathbf{u} \mathbf{u}^{T} V_{\varepsilon}^{-1} \\
& =I-\frac{2}{\mathbf{u}^{T} \varepsilon V_{\varepsilon}^{-1} \mathbf{u}} \mathbf{u u}^{T} \varepsilon V_{\varepsilon}^{-1} \\
& \rightarrow I-\frac{2}{\mathbf{u}_{1}^{T} W^{-1} \mathbf{u}_{1}}\left[\begin{array}{l}
\mathbf{u}_{1} \\
\mathbf{u}_{2}
\end{array}\right]\left[\mathbf{u}_{1}^{T} W^{-1} 0\right]
\end{aligned}
$$

as $\varepsilon \rightarrow 0$. Note that the action of this transformation can be considered to be on the scale defined by $\varepsilon$.

Numerical stability of the elementary $V$-invariant transformations depends on the size of the elements of $J$ and hence on its norm. In this connection the following result [2] is important.

Lemma 1. Let $J$ be an elementary reflector, and $\operatorname{set} \eta=\|\mathbf{u}\|\|\mathbf{v}\|>1$. Then the spectral norm of $J$ is given by

$$
\|J\|=\eta+\sqrt{ }\left\{\eta^{2}-1\right\}
$$

In the case of the $V$-invariant transformation (7)

$$
\eta=\frac{\|\mathbf{v}\|\|V \mathbf{v}\|}{\mathbf{v}^{T} V \mathbf{v}} .
$$

Thus the relative size of $\mathbf{v}^{T} V \mathbf{v}$ is important. 


\section{$3 \quad V$-Invariant Factorization}

The basic idea is to build up a $V$-invariant transformation $J$ taking $A$ to upper triangular form using elementary $V$-invariant transformations. Assume that a partial factorization $J_{i-1}$ has been constructed:

$$
J_{i-1} A=\left[\begin{array}{cc}
U_{i-1} & U_{12}^{i} \\
& A_{i}
\end{array}\right] .
$$

The aim now is to construct $\mathbf{v}$ such that the elementary $V$-invariant transformation (7)

$$
J^{i}=I-\frac{2}{\mathbf{v}^{T} V \mathbf{v}} V \mathbf{v} \mathbf{v}^{T}
$$

has the action

$$
J^{i}\left(J_{i-1} A \mathbf{e}_{i}\right)=J^{i}\left[\begin{array}{c}
\mathbf{u}_{i} \\
\mathbf{a}_{i}
\end{array}\right]=\left[\begin{array}{c}
\mathbf{u}_{i} \\
\gamma \mathbf{e}_{1}
\end{array}\right]
$$

giving

$$
J_{i}=J^{i} J_{i-1}, U_{i}=\left[\begin{array}{cc}
U_{i-1} & \mathbf{u}_{i} \\
& \gamma
\end{array}\right]
$$

Note that the scale of $\mathbf{v}$ is disposable. Thus the choice

$$
V \mathbf{v}=\left[\begin{array}{c}
0 \\
\mathbf{a}_{i}-\gamma \mathbf{e}_{1}
\end{array}\right]
$$

is allowed. This would be suitable except that computation of $\mathbf{v}$ requires the solution of a system of linear equations with matrix $V$, and this would seem to reintroduce exactly the kind of complication that we seek to avoid. There are considerable simplifications if $V_{2}$ is diagonal. In this case transformation of the design matrix to upper triangular form is made easier by some structural assumptions on $V$. These are that $V$ has the reduced form (5) where $V_{2} \in R^{n-k} \rightarrow R^{n-k}$, and

$$
V_{2}=\operatorname{diag}\left\{\nu_{k+1}, \nu_{k+2}, \cdots, \nu_{n}\right\}, 0<\nu_{k+1} \leq \nu_{k+2} \leq \cdots \leq \nu_{n} .
$$

If $V$ is a positive semidefinite matrix in general form then this could be achieved by

1. Scaling $V$ so that the diagonal elements of the transformed matrix are unity. Here $V \rightarrow S \bar{V} S$ where $S=\operatorname{diag}\left\{V_{11}^{1 / 2}, \cdots, V_{n n}^{1 / 2}\right\}$.

2. A diagonal pivoting (rank revealing) $L D L^{T}$ factorization [3] applied to $\bar{V}$ to construct a matrix of known factorization and rank which closely approximates $\bar{V}$

$$
P \bar{V} P=L D L^{T} .
$$

Note that the ordering achieved by this factorization gives the elements of $D$ in decreasing order of magnitude which is the inverse of that assumed in (5). 
3. Transformation of the problem to one with covariance matrix $D$

$$
\mathbf{r} \rightarrow L^{-1} P S^{-1} \mathbf{r} \Rightarrow A \rightarrow L^{-1} P S^{-1} A, \mathbf{b} \rightarrow L^{-1} P S^{-1} \mathbf{b} .
$$

The conditioning of the forward substitution is aided here by the use of diagonal pivoting which ensures that $\left|L_{i j}\right| \leq 1, j<i$. Thus illconditioning in $V$ tends to be concentrated in $D$ by the rank revealing factorization.

4. Permutation of the elements of the new covariance into increasing order with permutation matrix $Q$

$$
D \rightarrow Q D Q^{T}, \mathbf{r} \rightarrow Q \mathbf{r}
$$

To compute $\gamma$ let

$$
V_{2}=\left[\begin{array}{ll}
V_{11} & \\
& V_{22}
\end{array}\right], V_{11}=\operatorname{diag}\left\{\nu_{k+1}, \cdots, \nu_{i-1}\right\}, V_{22}=\operatorname{diag}\left\{\nu_{i}, \cdots, \nu_{n}\right\},
$$

where the partitioning is chosen to correspond to the i'th factorization step. Then

$$
J^{i}=\left[\begin{array}{lll}
I_{k} & & \\
& I_{1} & \\
& & I_{2}-\frac{2}{\mathbf{v}^{T} V_{22} \mathbf{v}} V_{22} \mathbf{v} \mathbf{v}^{T}
\end{array}\right]
$$

is $V$-invariant where $I_{k}$ is the $k \times k$ unit matrix and $I_{1}$ and $I_{2}$ are unit matrices conformable with $V_{11}$ and $V_{22}$ respectively. Then (11) gives

$$
\mathbf{v}=\left[\begin{array}{c}
0 \\
V_{22}^{-1} \mathbf{a}_{i}
\end{array}\right]
$$

The properties of the elementary reflector to give

$$
\begin{aligned}
{\left[\begin{array}{c}
\mathbf{u}_{i} \\
\mathbf{a}_{i}
\end{array}\right] } & =J^{i}\left[\begin{array}{c}
\mathbf{u}_{i} \\
\gamma \mathbf{e}_{1}
\end{array}\right], \\
& =\left(I-\frac{2}{\mathbf{v}^{T} V \mathbf{v}} V \mathbf{v} \mathbf{v}^{T}\right)\left[\begin{array}{c}
\mathbf{u}_{i} \\
\gamma \mathbf{e}_{1}
\end{array}\right] .
\end{aligned}
$$

Taking the scalar product with $\mathbf{v}$ gives a quadratic equation for $\gamma$

$$
\left(\mathbf{a}_{i}-\gamma \mathbf{e}_{1}\right)^{T} V_{22}^{-1}\left(\mathbf{a}_{i}+\gamma \mathbf{e}_{1}\right)=0 .
$$

This has the solution

$$
\gamma=\theta \sqrt{ }\left\{\nu_{i} \mathbf{a}_{i}^{T} V_{22}^{-1} \mathbf{a}_{i}\right\}
$$

where the standard argument which seeks to minimize cancellation suggests that $\theta=$ $-\operatorname{sgn}\left(\left(\mathbf{a}_{i}\right)_{1}\right)$ is appropriate. An interesting feature is the appearance of the term $N^{i}=$ $\nu_{i} V_{22}^{-1}$ which means that $\gamma$ is independent of the scale of $V$. [2] give the stability criterion

$$
\eta=\frac{\left\|V_{22}^{-1}\left(\mathbf{a}_{i}-\gamma \mathbf{e}_{1}\right)\right\|\left\|\mathbf{a}_{i}-\gamma \mathbf{e}_{1}\right\|}{\left(\mathbf{a}_{i}-\gamma \mathbf{e}_{1}\right)^{T} V_{22}^{-1}\left(\mathbf{a}_{i}-\gamma \mathbf{e}_{1}\right)} \geq \frac{\left\|\mathbf{a}_{i}\right\|}{2|\gamma|}
$$


Remark 2. Column pivoting can be used to exchange any column $\left[\begin{array}{l}\mathbf{u}_{j} \\ \mathbf{a}_{j}\end{array}\right], j=i, i+$ $1, \cdots, p$ of $\left[\begin{array}{c}U_{12}^{i} \\ A_{i}\end{array}\right]$ into the pivotal position. Let $\rho_{i}(A, j)=\mathbf{a}_{j}^{T} N^{i} \mathbf{a}_{j}$. The above analysis suggests that $j$ be chosen to maximize $\rho_{i}(A, j) /\left\|\mathbf{a}_{j}\right\|^{2}$ in order to make the lower bound for $\eta$ as small as possible. However, if $V \neq I$, lengths are not preserved by the $V$-invariant transformations and a consequence is that denominators cannot be updated economically in general. Thus the selection of the pivotal column would typically be made on the basis of the size of the $\rho_{i}(A, j)$ alone.

If $1 \leq l \leq k$ then the key mapping

$$
J \mathbf{a}_{l}=\gamma \mathbf{e}_{l}, l \leq k,
$$

needs to make use of the second family of $V$-invariant transformations (8). Let $\mathbf{a}_{1}=$ $\left[\begin{array}{l}\mathbf{a}_{1}^{1} \\ \mathbf{a}_{1}^{2}\end{array}\right]$ in conformity with the partitioning of $V$. Here the limiting argument leading to (9) provides insight into the reason why $V$-invariant transformations work well in the multiscaled situations. If $l=1$ the resulting transformation matrix $J=I-2 \mathbf{c d}^{T}$ has the generic form (8) with

$$
\begin{aligned}
\sqrt{2} \mathbf{c} & =\left(\mathbf{a}_{1}+\operatorname{sgn}\left(\left(\mathbf{a}_{1}^{1}\right)_{1}\right)\left\|\mathbf{a}_{1}^{1}\right\|_{2} \mathbf{e}_{1}\right) /\left\|\mathbf{a}_{1}^{1}\right\|_{2}, \\
\sqrt{2} \mathbf{d} & =\left[\begin{array}{c}
\mathbf{a}_{1}^{1}+\operatorname{sgn}\left(\left(\mathbf{a}_{1}^{1}\right)_{1}\right)\left\|\mathbf{a}_{1}^{1}\right\|_{2} \mathbf{e}_{1} \\
0
\end{array}\right] /\left(\left\|\mathbf{a}_{1}^{1}\right\|_{2}+\left|\left(\mathbf{a}_{1}^{1}\right)_{1}\right|\right) .
\end{aligned}
$$

This transformation will have large elements if

$$
\left\|\mathbf{a}_{1}^{1}\right\|_{2} \ll\left\|\mathbf{a}_{1}\right\|_{2} \text {. }
$$

This is the limiting case of (10) which characterizes illconditioning in the other class of transformations. The extreme case corresponds to $\left\|\mathbf{a}_{1}^{1}\right\|_{2}=0$, and this can certainly occur in the case $k>0$ in (5), (12). This is illustrated in the following example which provides a justification for the use of column pivoting in implementing the $V$-invariant factorization.

Example 1. Column pivoting is necessary for $V$-invariant solution methods if the generalised least squares problem is subject to equality constraints. Here the pivotal column at the i'th step is chosen as the one that maximizes $\rho_{i}(A, j)=\sum_{q=i}^{n+m} N_{q}^{i} A_{q j}^{2}, j=$ $i, i+1, \cdots, p$, where $m$ is the number of equality constraints and $N^{i}$ is the scaled diagonal weighting matrix. To illustrate the requirement consider the design matrix $A_{2} \in R^{p} \rightarrow R^{n}$ given by

$$
\begin{aligned}
\left(A_{2}\right)_{* 1} & =S \mathbf{e}, \\
\left(A_{2}\right)_{i(2 j)} & =S_{i} \cos (2 \pi j(i-1) h), \\
\left(A_{2}\right)_{i(2 j+1)} & =S_{i} \sin (2 \pi j(i-1) h), \\
i=1,2, \cdots, n, j & =1,2, \cdots, k,
\end{aligned}
$$


where $p=2 k+1, S=\operatorname{diag}\{1 / \sqrt{2}, 1, \cdots, 1,1 / \sqrt{2}\}, h=1 /(n-1)$. The columns of $A_{2}$ are orthogonal and similarly scaled so a least squares problem with $A_{2}$ as design is very well conditioned. Let the rows of the constraint matrix $A_{1} \in R^{p} \rightarrow R^{2}$ be given by

$$
\left(A_{1}\right)_{1 *}=\mathbf{e}^{T},\left(A_{1}\right)_{2 *}=\mathbf{e}^{T}-p \mathbf{e}_{k+1}^{T} .
$$

Then the constrained least squares problem

$$
\min _{\mathbf{s}, \mathbf{x}} \mathbf{s}^{T} \mathbf{s} ;\left[\begin{array}{c}
0 \\
I
\end{array}\right] \mathbf{s}=\left[\begin{array}{l}
A_{1} \\
A_{2}
\end{array}\right] \mathbf{x}-\left[\begin{array}{l}
\mathbf{b}_{1} \\
\mathbf{b}_{2}
\end{array}\right],
$$

with

$$
\left[\begin{array}{l}
\mathbf{b}_{1} \\
\mathbf{b}_{2}
\end{array}\right]=\left[\begin{array}{c}
A_{1} \mathbf{e} \\
A_{2} \mathbf{e}+\varepsilon
\end{array}\right], \varepsilon_{i}=\sin (2 \pi(k+1)(i-3) h), i=3,4, \cdots, n+2,
$$

has the exact solution $\mathbf{x}=\mathbf{e}$. However, the leading $2 \times 2$ submatrix of $A_{1}$ is singular if $k \geq 2$. This causes a breakdown of the $V$-invariant factorization at the second step (13) as a consequence of the form of $N^{2}$ if column interchanges are not used. In the particular case corresponding to $n=8, p=5$, the ordering resulting from column pivoting is $\{3,2,1,4,5\}$. The first interchange is a consequence of the largest coefficient in the second constraint, and it succeeds in forcing the leading $2 \times 2$ submatrix to be nonsingular.

\section{Does the Use of the $L D L^{T}$ Factorization of $V$ Make Sense?}

In this section it is assumed that $V$ has full rank, but is illconditioned as a result of a cluster of small eigenvalues. The results extend immediately to the case where $V$ has a known nullspace plus a cluster of small eigenvalues. Here the rank-revealing Cholesky factorization of $V$, performed in exact arithmetic, has the form

$$
P V P^{T} \rightarrow L \operatorname{diag}\left\{D_{n}, D_{n-1}, \cdots, D_{1}\right\} L^{T}
$$

where $P$ is the permutation matrix corresponding to the diagonal pivoting, and the diagonal pivoting ensures that

$$
D_{n} \geq D_{n-1} \geq \cdots \geq D_{1} .
$$

This order is the reverse of that required here, and it must be inverted in order to construct the factorization of the design matrix based on $V$-invariant transformations. Conditions which guarantee that $n-k$ steps of the Cholesky factorization with diagonal pivoting can be computed leaving a small remainder are given in [3]. One form compatible with the above assumption is

$$
\begin{aligned}
& \Delta_{1}=\operatorname{diag}\left\{D_{1}, D_{2}, \cdots, D_{k}\right\}>0, \text { small, } \\
& D_{k} \ll D_{k+1}, \\
& \Delta_{2}=\operatorname{diag}\left\{D_{k+1}, \cdots, D_{n}\right\} \text { not small, } \\
& \text { where } k \leq p
\end{aligned}
$$


It would be expected that if the computed factorization does go to completion then the computed values $\left\{D_{1}, D_{2}, \cdots, D_{k}\right\}$ could have high relative error as a result of cancellation. Does this matter? The following argument suggests strongly that it does not. It assumes that the factorization is stopped after $n-k$ steps. This yields the incomplete transformation

$$
P V P^{T}=L\left[\begin{array}{ll}
\Delta_{2} & \\
& V^{k}
\end{array}\right] L^{T},
$$

where use has been made of the result that the unit lower triangular matrix $L$ has the particular structure

$$
L=\left[\begin{array}{cc}
L_{n-k} & \\
L_{2}^{(n-k)} & I
\end{array}\right],
$$

and

$$
L^{-1}\left[\begin{array}{cc}
0 & 0 \\
0 & V^{k}
\end{array}\right] L^{-T}=\left[\begin{array}{cc}
0 & 0 \\
0 & V^{k}
\end{array}\right] .
$$

First note that the case $D=\operatorname{diag}\left\{0, \cdots, 0, D_{k+1}, \cdots, D_{n}\right\}$ corresponds to the equality constrained problem

$$
\min _{\mathbf{x}} \mathbf{s}^{T} \mathbf{s} ;\left[\begin{array}{ll}
0 & \\
& \Delta_{2}^{1 / 2}
\end{array}\right] \mathbf{s}=\left[\begin{array}{l}
A_{1} \\
A_{2}
\end{array}\right] \mathbf{x}-\left[\begin{array}{l}
\mathbf{b}_{1} \\
\mathbf{b}_{2}
\end{array}\right] .
$$

Here the Cholesky $L$ has been absorbed into the design to simplify notation $\left(\mathbf{r} \rightarrow L^{-1} \mathbf{r}\right)$, and it is assumed that necessary permutations to order $D$ have also been applied. This is the limiting problem as $\lambda \rightarrow \infty$ associated with the penalised objective

$$
\min _{\mathbf{x}}\left\{\mathbf{r}_{2}^{T} \Delta_{2}^{-1} \mathbf{r}_{2}+\lambda \mathbf{r}_{1}^{T} E^{-1} \mathbf{r}_{1}\right\} ; \mathbf{r}=\left[\begin{array}{l}
A_{1} \\
A_{2}
\end{array}\right] \mathbf{x}-\left[\begin{array}{l}
\mathbf{b}_{1} \\
\mathbf{b}_{2}
\end{array}\right]
$$

where $\lambda^{-1} E=V^{k}$ where $V^{k} \in R^{k} \rightarrow R^{k}$ is small as the remainder after $n-k$ steps of the rank-revealing Cholesky factorization [3] p. 212, $E$ is positive definite, and $\lambda=1 /\left\|V^{k}\right\|$. The problem (14) has the alternative form

$$
\min _{\mathbf{x}} \mathbf{s}^{T} \mathbf{s} ;\left[\begin{array}{ll}
\lambda^{-1 / 2} E^{1 / 2} & \\
& \Delta_{2}^{1 / 2}
\end{array}\right] \mathbf{s}=\left[\begin{array}{l}
A_{1} \\
A_{2}
\end{array}\right] \mathbf{x}-\left[\begin{array}{l}
\mathbf{b}_{1} \\
\mathbf{b}_{2}
\end{array}\right] .
$$

Now penalty function theory can be used to show $\|\mathbf{x}(\lambda)-\widehat{\mathbf{x}}\|=O(1 / \lambda), \lambda \rightarrow \infty$ provided

$$
M=\left[\begin{array}{cc}
A_{2}^{T} \Delta_{2}^{-1} A_{2} & A_{1}^{T} E^{-T / 2} \\
E^{-1 / 2} A_{1} & 0
\end{array}\right]
$$

has full rank [1]. This is a weaker condition than both $A_{1}$ and $A_{2}$ having full rank. Here the conditioning of the scaled perturbation matrix $E$ could also be important. Assuming that $E$ is reasonably well conditioned then the equality constrained problem obtained 
by setting $\Delta_{1}=0$ has a well defined solution which differs from the exact solution of that based on the computed $L D L^{T}$ factorization by $O\left(\left\|\Delta_{1}\right\|\right)$.

The above argument identifies a class of problems where $V$ is illconditioned for inversion, but where solution by $V$-invariant methods is satisfactory after preliminary problem transformation based on the $L D L^{T}$ factorization of $V$ with diagonal pivoting . One relevant case is the fourth example in [4]. This is defined to have an illconditioned $L$, and unit diagonal. However, if the $L D L^{T}$ factorization is recomputed using diagonal pivoting then the illconditioning is moved to $D$ and does not cause problems.

\section{References}

1. A.V. Fiacco and G.P. McCormick, Nonlinear programming: Sequential unconstrained minimization techniques, John Wiley and Sons, Inc., 1968.

2. M. Gulliksson and P. Wedin, Modifying the QR-decomposition to constrained and weighted linear least squares, SIAM J. Matrix Anal. Appl. 13 (1992), no. 4, 1298--1313.

3. N.J. Higham, Accuracy and stability of numerical algorithms, SIAM, 1996.

4. I. Søderkvist, On algorithms for generalized least squares problems with ill-conditioned covariance matrices, Computational Statistics 11 (1996), 303-313. 